\title{
Predicting factors of symptomatic radiation pneumonitis induced by durvalumab following concurrent chemoradiotherapy in locally advanced non-small cell lung cancer
}

Hiroshi Mayahara ${ }^{1 *} \mathbb{0}$, Kazuyuki Uehara ${ }^{1}$, Aya Harada ${ }^{1}$, Keiji Kitatani ${ }^{1}$, Tomonori Yabuuchi ${ }^{1}$, Shuichirou Miyazaki ${ }^{1}$, Takeaki Ishihara ${ }^{2}$, Hiroki Kawaguchi ${ }^{2}$, Hikaru Kubota ${ }^{2}$, Hideaki Okada ${ }^{3}$, Taira Ninomaru ${ }^{3}$, Chihiro Shindo ${ }^{4}$ and Akito $\mathrm{Hata}^{3}$

\begin{abstract}
Background: Concurrent chemoradiotherapy (CCRT) followed by durvalumab is the standard of care for unresectable locally-advanced non-small cell carcinoma (LA-NSCLC). However, a major concern about administration of durvalumab after CCRT is whether the incidence of symptomatic radiation pneumonitis (RP) may increase or not. In the present analysis, we report the initial results of CCRT followed by durvalumab in patients with LA-NSCLC in a realworld setting with focus on predicting factors for symptomatic RP.

Methods: Patients who were pathologically diagnosed as NSCLC and initiated treatment with CCRT followed by durvalumab between July 2018 to December 2019 were eligible for this study. Patients were included if they completed the planned CRT course and administered at least one course of durvalumab. We retrospectively investigated the preliminary survival outcome and incidence and predicting factors for symptomatic RP.

Results: Of the 67 patients who planned CCRT, 63 patients completed the entire CCRT course. Of these, 56 patients proceeded to consolidation with durvalumab. The median time to eternal discontinuation of durvalumab was 9.7 months. The cumulative proportion of the patients who exhibited symptomatic RP was 30,40 and $44 \%$ at 3,6 and 12 months, respectively. In multivariate analyses, pulmonary fibrosis score and lung V40 were significant predictive factors for symptomatic RP ( $p<0.001$, HR: 7.83, 95\% Cl: 3.38-18.13, and $p=0.034$, HR: 3.17, 95\% Cl: 1.09-9.19, respectively).
\end{abstract}

Conclusions: Pulmonary fibrosis sore and lung V40 were significant predictive factors for symptomatic RP. We should be cautious about the administration of durvalumab for patients having subclinical pulmonary fibrosis. To our best knowledge, this is one of the first report showing the predictive value of high dose volumes to the lung in patients with LA-NSCLC who received CCRT followed by durvalumab.

Keywords: Locally advanced non-small cell lung cancer, Concurrent chemoradiotherapy, Radiation pneumonitis, Dosimetric factor, Durvalumab

\footnotetext{
*Correspondence: mayahara@k-mcc.net

${ }^{1}$ Department of Radiation Oncology, Kobe Minimally-invasive Cancer Center, 8-5-1, Minatojima-Nakamachi, Chuo-Ku, Kobe, Hyogo 650-0046, Japan

Full list of author information is available at the end of the article
}

\section{Introduction}

Lung cancer is the most frequently diagnosed cancer and is the leading cause of cancer mortality, world widely [1]. Locally advanced Stage 3 non-small cell lung cancer original author(s) and the source, provide a link to the Creative Commons licence, and indicate if changes were made. The images or other third party material in this article are included in the article's Creative Commons licence, unless indicated otherwise in a credit line to the material. If material is not included in the article's Creative Commons licence and your intended use is not permitted by statutory regulation or exceeds the permitted use, you will need to obtain permission directly from the copyright holder. To view a copy of this licence, visit http://creativecommons.org/licenses/by/4.0/. The Creative Commons Public Domain Dedication waiver (http://creativeco mmons.org/publicdomain/zero/1.0/) applies to the data made available in this article, unless otherwise stated in a credit line to the data. 
(LA-NSCLC) accounts for $20 \%$ of lung cancer cases [2]. Because of frequency in both of locoregional and distant recurrences, concurrent chemoradiotherapy (CCRT) has long been a standard of care for decades $[3,4]$. The 5 -year overall survival (OS) ratio has estimated to be only $15-30 \%$ [5-10]. Numerous studies had tested combination of new systemic agents or dose escalation and failed to improve outcomes $[6,8,9,11,12]$. Several studies investigated consolidative chemotherapy after CCRT and showed no apparent clinical benefit [13-17].

The PACIFIC phase 3 randomized controlled trial demonstrated efficacy of consolidation therapy with durvalumab [18-20]. Durvalumab is a selective human IgG1 monoclonal antibody that blocks programmed death ligand-1(PD-L1) binding to PD-1 receptor and CD80, and it increases the anti-tumor activity by $\mathrm{T}$ cells [2123]. In the PACIFIC study, for patients with LA-NSCLC, durvalumab administered after CCRT improved median Progression-free survival (PFS) by 17.2 months compared to its placebo of 5.6 months. The median OS was 47.5 months with durvalumab but was 29.1 months with placebo [20]. Now, administration of durvalumab after CCRT has become to be a standard of care [24].

A major concern about administration of durvalumab after CCRT is whether the incidence and severity of radiation pneumonitis (RP) may increase or not. In the PACIFIC study, RP was observed in $34 \%$ and $25 \%$ of the patients with durvalumab and placebo, respectively [18]. In particular, grade 3 and 4 RP occurred in $3.4 \%$ and 2.6 $\%$ of patients with durvalumab and placebo, respectively. In the PACIFIC study, patients were randomly assigned to groups after the successful completion of CCRT and those who exhibited symptomatic RP during and immediately after the CCRT were excluded from study inclusion [18]. The reported incidence of RP in the PACIFIC study may not represent a real-world incidence, because it might include only well-conditioned patients. Additionally, actual dosimetric factors, such as lung dose, target coverage, irradiation techniques, or quality of radiotherapy plans were not evaluated, because the part of CCRT was not included in the protocol of the PACIFIC study [18].

In the present analysis, we report the results of CCRT followed by durvalumab in patients with unresectable LA-NSCLC in a real-world setting with focus on predicting factors for symptomatic RP.

\section{Materials and methods Study subjects}

Patients with either unresectable primary LA-NSCLC and locoregional recurrent NSCLC after primary resection were included in this study. Patients who were pathologically diagnosed as NSCLC and initiated treatment with CCRT followed by durvalumab between July 2018 to December 2019 were eligible for this study. The data cutoff date was August 31, 2020.

\section{Patient characteristics}

Fifteen-six patients with LA-NSCLC who completed CCRT and received maintenance therapy with durvalumab were eligible for this analysis. Patients' baseline characteristics are summarized in Table 1 . Between July 2018 and December 2019, a total of 78 patients received definitive radiotherapy in our single institution. Among them, 63 were with unresectable primary LA-NSCLC

Table 1 Patients' characteristics

\begin{tabular}{|c|c|}
\hline Characteristics & $\mathrm{N}=56(\%)$ \\
\hline \multicolumn{2}{|l|}{ Age } \\
\hline Median years (range) & $72(48-85)$ \\
\hline \multicolumn{2}{|l|}{ Gender } \\
\hline Male/female & $37(66) / 19(34)$ \\
\hline \multicolumn{2}{|l|}{ Performance status } \\
\hline $0 / 1 / 2$ & $24(43) / 28(50) / 4(7)$ \\
\hline \multicolumn{2}{|l|}{ Smoking status } \\
\hline Current / Former / Never & $20(36) / 25(45) / 11(20)$ \\
\hline \multicolumn{2}{|l|}{ Histology } \\
\hline Adeno / Sq / Non-small & $25(45) / 30(54) / 1(2)$ \\
\hline \multicolumn{2}{|l|}{ Primary tumor location } \\
\hline $\begin{array}{l}\text { Upper lobe or trachea / Middle or } \\
\text { lower lobe }\end{array}$ & $33(59) / 23(41)$ \\
\hline \multicolumn{2}{|l|}{ Clinical stage } \\
\hline||$|\mathrm{A} /||\mathrm{B} /||| \mathrm{C} /$ Others & $19(34) / 14(25) / 10(18) / 13(23)$ \\
\hline \multicolumn{2}{|l|}{ PD-L1 Satus } \\
\hline$\geq 50 \% / 1-49 \% /<1 \% /$ unknown & $9(16) / 11(20) / 19(24) / 17(30)$ \\
\hline \multicolumn{2}{|l|}{ Irradiation technique } \\
\hline IMRT & $28(50)$ \\
\hline 3D-CRT & $28(50)$ \\
\hline \multicolumn{2}{|l|}{ Total radiotherapy dose } \\
\hline $60 \mathrm{~Gy} / 30 \mathrm{fr}$ & $48(86)$ \\
\hline $66 \mathrm{~Gy} / 33 \mathrm{fr}$ & $5(9)$ \\
\hline $54 \mathrm{~Gy} / 27 \mathrm{fr}$ & $1(2)$ \\
\hline $50 \mathrm{~Gy} / 25 \mathrm{fr}$ & $2(4)$ \\
\hline \multicolumn{2}{|l|}{ Chemotherapy regimen } \\
\hline$w C B D C A+P T X$ & $26(46)$ \\
\hline$C D D P+V N R$ & $12(21)$ \\
\hline CDDP + Pemetrexed & $12(21)$ \\
\hline $\mathrm{CDDP}+\mathrm{S}-1$ & $6(11)$ \\
\hline \multicolumn{2}{|l|}{ Pulmonary function test } \\
\hline Median \%VC (range) & $89.0(53.5-124.4)$ \\
\hline Median \%FEV1.0 (range) & $80.6(46-126)$ \\
\hline
\end{tabular}

Adeno adenocarcinoma, $S q$ squamous cell carcinoma, $P D-L 1$ programmes cell death -ligand 1, IMRT intensity modulated radiation therapy, $3 D-C R T$ three dimensional-conformal radiation therapy, fr fractions, $w$ weeekly, CBDCA carboplatin, $P T X$ paclitaxel, CDDP cisplatin, VNR vinorelbine, $V C$ vital capacity, FEV forced expiratory volume 
and 15 were with unresectable locoregional recurrent NSCLC after primary resection. Excluding 12 patients who were planned to be treated with radiotherapy alone, 67 patients were planned to receive CCRT. Sixty-three patients completed planned CCRT course, whereas 4 patients discontinued CCRT because of massive respiratory bleeding, tracheoesophageal fistula, chemotherapy-induced pneumonitis, and patient's refusal for chemotherapy, respectively. Excluding these 4 patients, 63 patients completed CCRT. Of these, 56 patients received durvalumab after a median of 19 days from the last day of irradiation. Seven patients did not receive durvalumab, due to surgical resection in 2 , comorbidity in 2 , early symptomatic RP in 1 , deteriorated performance status in 1 and patient's refusal in 1, respectively. These patients were excluded from further analysis to maintain comparability with the results of PACIFIC study. Thus, 56 of $67(84 \%)$ patients who planned CCRT proceeded to maintenance therapy with durvalumab. Applied irradiation techniques were intensity modulated radiotherapy (IMRT) for 28 patients and 3D-conformal radiation therapy (3D-CRT) for 28 patients.

The patient's consent for the treatment was obtained in a written form. Clinical staging was done by fluorodeoxyglucose-positron emission tomography, contrast-enhanced computed tomography (CT) and gadolinium-enhanced magnetic resonance imaging (MRI) of the brain, according to the Union for International Cancer Control criteria (8th ed.). Patients were included if they completed the planned CRT course and administered at least one course of durvalumab. We retrospectively investigated the incidence and predicting factors for symptomatic RP. This study was approved by our Institutional Review Board and was conducted in accordance with the Declaration of Helsinki.

\section{Statistical analysis}

The primary objective of this analysis was to describe the clinical outcomes associated with CCRT followed by durvalumab. OS and PFS was estimated as the time from starting CCRT to death or disease progression, by using the Kaplan-Meier method.

Possible clinical and dosimetric factors that may predict symptomatic RP were statistically investigated. Symptomatic RP was defined as Grade 2 or higher RP (G2RP) by the Common Toxicity Criteria for Adverse Events (Version 5.0). The time to G2RP was defined as the time from completion of CCRT to the development of G2RP and was calculated by using a Kaplan-Meier estimator, and compared by using a log-rank test. Time to discontinue durvalumab (TTDD) was defined as the time from the first administration of durvalumab to 14 days after the last administration of durvalumab. Temporary
Table 2 Definition of pulmonary fibrosis score

\begin{tabular}{ll}
\hline Score & Definition \\
\hline 0 & No fibrosis \\
1 & Interlobular septal thickening; no descrete honeycoming \\
2 & Honeycoming (with or without septal thickening) involv- \\
& ing $<25 \%$ of the lobe \\
3 & Honeycoming involving $25-49 \%$ of the lobe \\
5 & Honeycoming involving $50-75 \%$ of the lobe \\
\hline
\end{tabular}

postponement of durvalumab due to toxicity, or completion after 12 month of administration was not counted for an event. Disease progression and discontinuation of durvalumab by the reason other than RP were treated as competing risk for TTDD due to RP, and the hazard ratio (HR) was estimated using the Fine-Gray method.

The percent of lung volumes receiving above various dose levels were statistically evaluated. The parameters assessed included percentage of total lung volume (lung minus gross tumor volume) exceeding 50Gy (V50), 40Gy (V40), 30Gy (V30), 20Gy(V20), 10Gy (V10), 5Gy (V5), mean lung dose (MLD), volume of the lung received less than 5 Gy (Vs5) and initial planning target volume (PTV). For detecting optimal cut-off values of continuous variables, we underwent receiver-operating characteristic (ROC) analyses, and the optimal cut-off values were determined by Youden index. Then, areas under the curve (AUC) were calculated for each value. Associations between dosimetric variables were evaluated by using the Pearson correlation coefficient. A correlation coefficient of more than 0.6 was regarded as having some correlation between variables. When we faced with factors that were correlated with each other, we selected the factor that had the highest area under the curve (AUC) in ROC analyses. Multivariate analyses by using Fine-Gray model were performed including factors that had shown significant associations $(p<0.05)$ in univariate Gray's test.

All analyses were performed in $R$, version 3.6.3 ( $R$ Foundation for Statistical Computing). All hypothesis tests were 2 -sided and a $p<.05$ was considered statistically significant.

\section{Image analysis}

In regard to the evaluation of baseline lung fibrosis, we used pulmonary fibrosis score, which was declared by Kazerooni EA, et al and modified by Tsujino et al. [25, 26]. Pulmonary fibrosis was scored according to the extent of the subpleural focal honeycombing. The scoring definition of pulmonary fibrosis is shown in Table 2. Pulmonary fibrosis scores were independently reviewed by an experienced diagnostic radiologist, pulmonary 
medical oncologist and radiation oncologist, those who were blinded from patient's medical records. If there was any discordance in an evaluation in pulmonary fibrosis score, the score was decided on discussion among them. Interreader agreement analysis was not performed.

\section{Radiotherapy}

Radiotherapy was delivered using a 10 or 6-MV Xray by TrueBeam (Varian Medical Systems, CA, USA). Fourdimensional CT (4-DCT) was used to evaluate respiratory tumor motion. Varian's RPM respiratory-gating irradiation system was used if the respiratory tumor motion encompasses $10 \mathrm{~mm}$. For dose calculation, images of expiratory phase (a $2 \mathrm{~mm}$ thickness) were used. The Eclipse (ARIA 11.0.42, Varian Medical Systems, CA, USA) treatment planning software was used for dose optimization and calculation. Irradiation techniques included both of IMRT and 3D-CRT. The irradiation technique was decided at the discretion of the attending radiation oncologist, in consideration of the anatomical tumor location, tumor extension and treatment schedule. All the irradiations were delivered under image guidance by orthogonal on-board imager (OBI) and $\mathrm{kV}$ cone beam CT (CBCT). Gross target volume (GTV) of the primary lesion was defined in simulated CT images of the lung window. Internal target volume (ITV) was determined by the summation of GTVs in 4-D CT images to encompass whole respiratory tumor motion. In case of respiratorygating, ITV was determined as summation of GTVs in only end-respiratory phase (typically, $40-60 \%$ of the respiratory cycle). Clinical target volume (CTV) included a $5 \mathrm{~mm}$ margin in all directions from ITV. Prophylactic regional irradiation was basically not applied. A PTV was defined as CTV with a 4-5 $\mathrm{mm}$ margin to compensate for any set-up error. Prescribed dose was 60 Gy in 30 fractions for all the patients, except for one case who discontinued irradiation at a dose of 54 Gy in 27 fractions, due to infectious pneumonitis. The dose was prescribed to an isocenter in a case for 3D-CRT, whereas the dose was prescribed to D50\% of the PTV in a case for IMRT until April 2019. Then it was switched to D95\% of the PTV thereafter, in accordance with protocol of another prospective observational clinical study. Dose constraints for organs at risk were $<45$ Gy to spinal cord and V20, V5 of the lung should be $<30 \%,<65 \%$, respectively.

\section{Chemotherapy}

The concurrent chemotherapy regimens included weekly carboplatin + paclitaxel (PTX), cisplatin (CDDP) + S -1 , $\mathrm{CDDP}+$ vinorelbine and CDDP + pemetrexed. The regimen was determined at the discretion of the attending medical oncologists depending on the patients' age, general condition, organ functions and tumor histology.

\section{Durvalumab}

Diagnostic CTs were taken immediately after completing CCRT to evaluate its efficacy and to detect RP. If no abnormalities were found on $\mathrm{CT}$ and blood tests, durvalumab was started. Durvalumab $(10 \mathrm{mg} / \mathrm{kg})$ was administered intravenously every 2 weeks until 1 year [18]. The administration of durvalumab was continued until disease progression, emergence of unacceptable toxicities such as G2RP or withdrawal of consent. If patients developed G2RP, they typically were treated by corticosteroids with prednisolone of $0.5-1.0 \mathrm{mg} / \mathrm{kg}$, and the administration of durvalumab were postponed until they resolved the symptom and reduced prednisolone to a dose of less than 5-10mg per body.

\section{Follow-up}

After starting durvalumab, patients were suggested to receive chest X-ray and blood test for every bi-weekly visit for durvalumab. Chest and upper abdominal CT images were taken for every 2 months for the first year, every 3-4 months thereafter. Brain MRI were taken for every 6 months.

\section{Results}

\section{OS, PFS and cause of morbidity}

With a median follow-up period of 14.0 months for the living patients, the 12- and 18-months OS ratio were 87 and $84 \%$, respectively (Fig. 1a). At the time of analysis, 9 patients had deceased. Six of them had died from primary disease progression, 1 from another cancer and 2 from treatment-related toxicities (lung toxicity in one and toxic epidermal necrolysis in one). The 12- and 18-months PFS were 57 and 46\%, respectively (Fig. 1b).

\section{Continuity of durvalumab}

At the time of analysis, 19 patients completed 1 year of durvalumab administration, whereas 8 patients were currently under administration. Twenty-nine patients discontinued durvalumab. Of these, 15 discontinued durvalumab due to disease-progression, 11 by toxicity, and 3 from patient's refusal. The proportion of the patients who were continuing durvalumab at 3, 6 and 12 months was 70, 63 and 48\%, respectively (Fig. 2). The median TTDD was 9.7 months.

\section{Incidence of radiation pneumonitis}

The number of the patients who developed RP of Grade $0,1,2,3$ and 5 were 6 (10.7\%), 28 (50\%), 17 (30.4\%), 4 (7.1\%) and $1(1.8 \%)$, respectively. Case presentations on the typical clinical courses of radiation pneumonitis are available in the Additional file 1: Appendix 1 and 2. The cumulative proportion of the patients who exhibited G2RP was 30, 40 and $44 \%$ at 3, 6 and 12 months, 

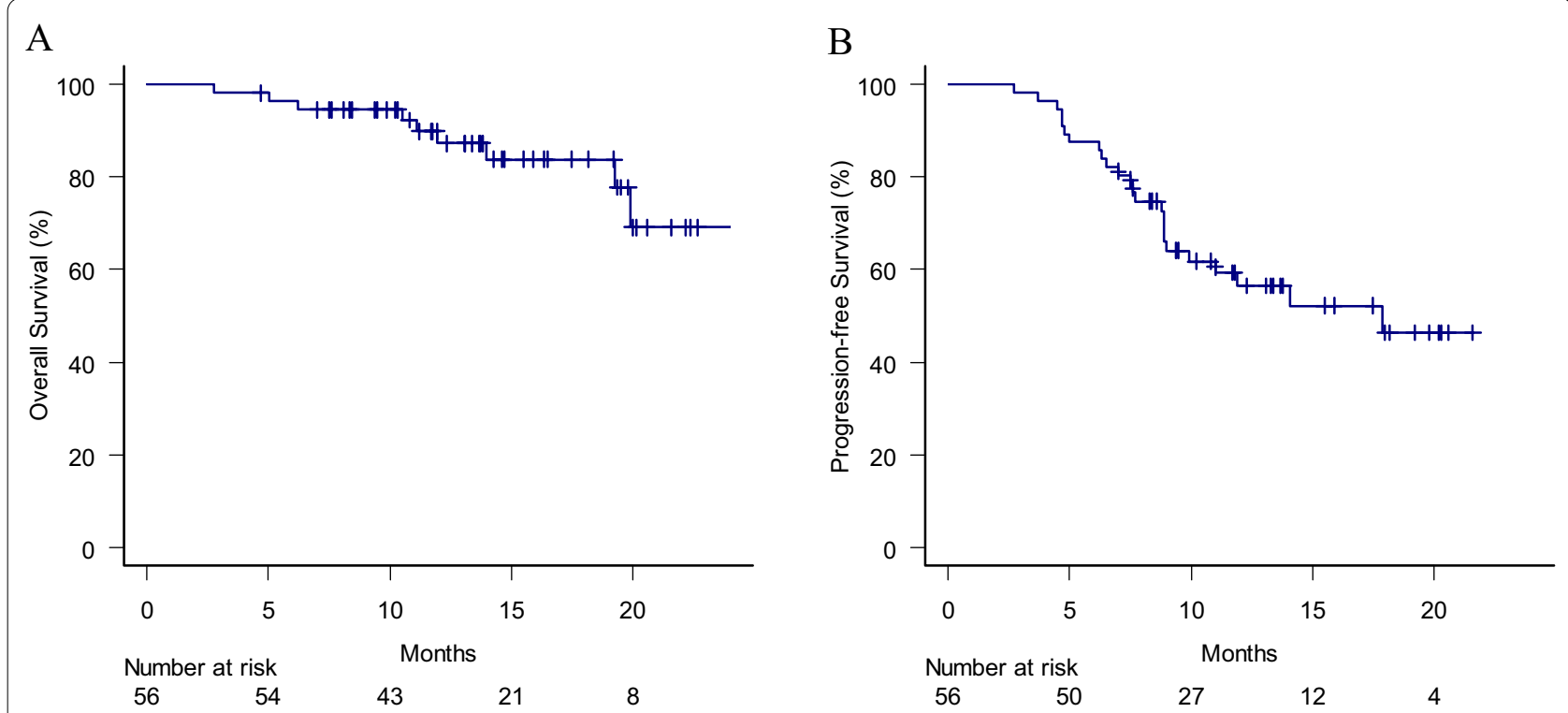

Fig. 1 a Kaplan-Meier curve for overall survival of the eligible patients. b Kaplan-Meier curve for progression-free survival of the eligible patients

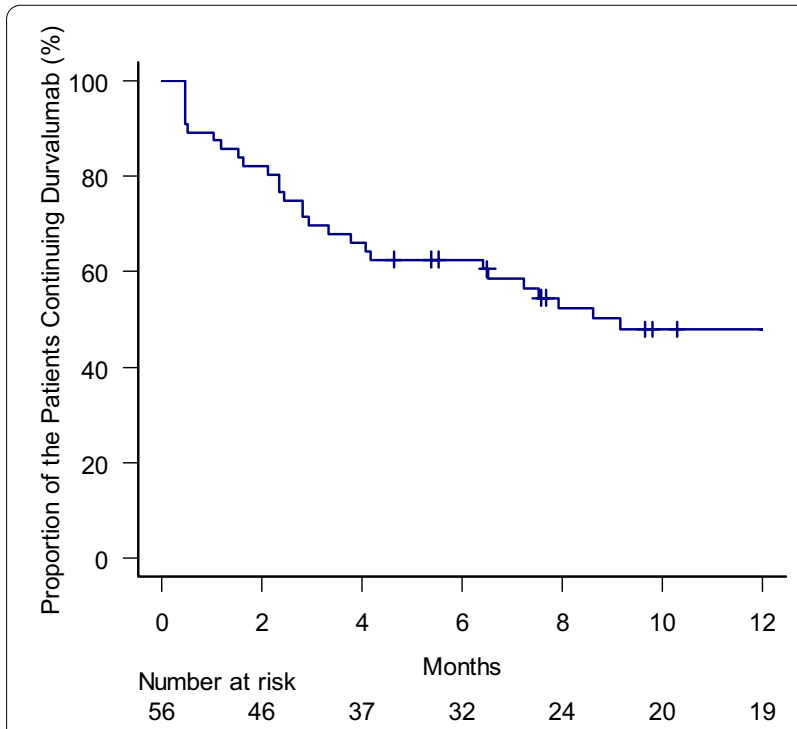

Fig. 2 Kaplan-Meier curve for proportion of the patients who were continuing durvalumab

respectively (Fig. 3). Oral prednisolone of $0.5-1.0 \mathrm{mg} /$ $\mathrm{kg}$ was administered to 19 out of the 22 patients with G2RP. Six patients resumed durvalumab after the remission of RP. The proportion of the patients who eternally discontinued durvalumab due to G2RP was 14,14 and $14 \%$ at 3,6 and 12 months, respectively (Fig. 4).

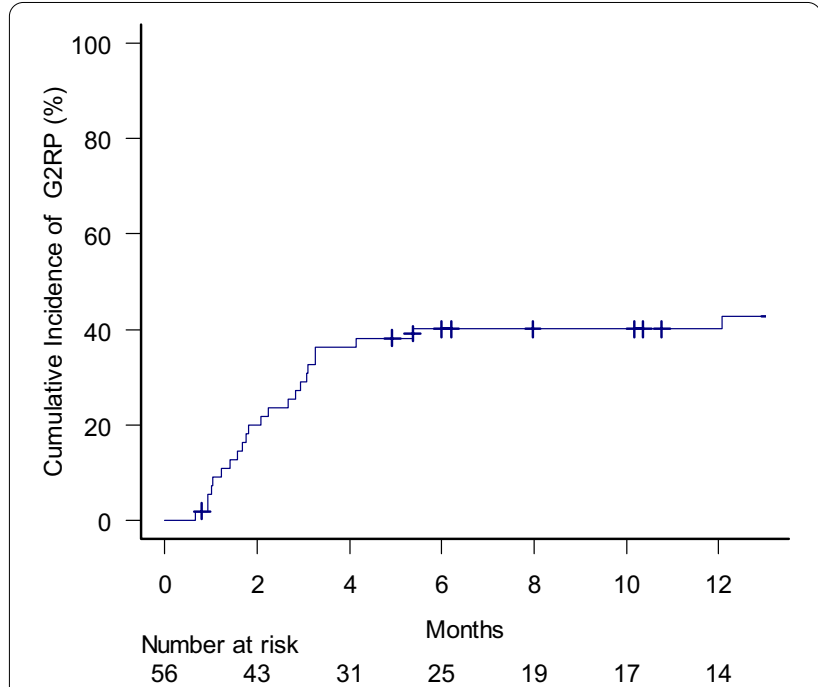

Fig. 3 Cumulative incidence of radiation pneumonitis of grade 2 or more

ROC analysis of the dose-volume histogram parameters of the lung for G2RP

The results of ROC analysis for G2RP are summarized in Table 3 . The patients were dichotomized according to the threshold levels determined by the ROC analysis. Cumulative incidences of G2RP were estimated by the Gray's test. Discontinuation of durvalumab due to the cause other than G2RP was treated as a competing risk for G2RP. Lung V30, V40, V50, mean lung dose (MLD) and initial planning target volume (PTV) 


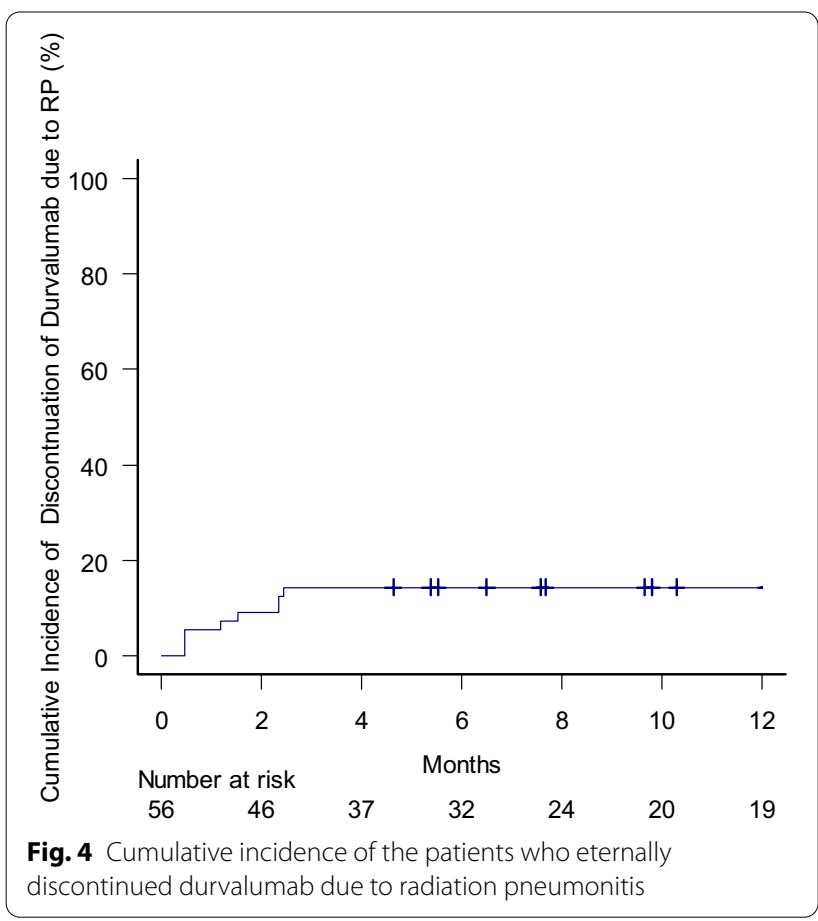

Table 3 ROC analysis of the dose-volume histogram parameters of the lung and incidence of radiation pneumonitis of grade 2 or more

\begin{tabular}{|c|c|c|c|c|c|}
\hline \multirow[t]{2}{*}{ Dose (Gy) } & \multirow[t]{2}{*}{ AUC } & \multirow[t]{2}{*}{$\begin{array}{l}\text { Threshold } \\
\text { level (\%) }\end{array}$} & \multicolumn{2}{|c|}{$\begin{array}{l}\text { Cumulative incidence of } \\
\text { G2RP at } 6 \text { months }\end{array}$} & \multirow[t]{2}{*}{$p$ value } \\
\hline & & & $\geq$ threshold & $<$ threshold & \\
\hline Lung V50 & 0.640 & 5.3 & 56.8 & 13.6 & 0.008 \\
\hline Lung V40 & 0.686 & 10.0 & 57.3 & 16.7 & 0.011 \\
\hline Lung V30 & 0.644 & 15.7 & 57.3 & 26.1 & 0.048 \\
\hline Lung V20 & 0.608 & 23.0 & 56.4 & 29.8 & 0.16 \\
\hline Lung V10 & 0.566 & 34.1 & 52.9 & 30.0 & 0.12 \\
\hline Lung V5 & 0.570 & 48.2 & 55.0 & 30.5 & 0.07 \\
\hline $\begin{array}{l}\text { Mean lung } \\
\text { dose }\end{array}$ & 0.640 & 12.1 & 54.0 & 21.1 & 0.046 \\
\hline Lung Vs5 (ml) & 0.533 & 1364 & 41.1 & 37.6 & 0.62 \\
\hline $\begin{array}{l}\text { Initial PTV } \\
(\mathrm{ml})\end{array}$ & 0.679 & 398 & 58.3 & 23.8 & 0.024 \\
\hline
\end{tabular}

$R O C$ receiver-operating characteristic, G2RP radiation pneumonitis of grade2 or more, Lung Vx percentage of the lung volume exceeding $x$ Gy, Lung Vs5 volume of the lung received less than $5 \mathrm{~Gy}$, PTV planning target volume

were significant predictors for G2RP. Lung V20, V10 and V5 did not significantly predicted G2RP. Pearson correlation coefficients between Lung V30/V40, V40/ $\mathrm{V} 50$ and V30/V50 were $0.730,0.853$, and 0.629 , respectively. Pearson correlation coefficients between MLD/ V30, MLD/V40 and MLD/V50 were 0.762, 0.802, and 0.661 , respectively. Because lung V30, V40, V50 and
MLD were correlated with each other, we selected lung V40, which had highest AUC among them, for further analysis.

\section{Univariate and multivariate analyses of factors affecting risk of G2RP}

Univariate analyses for G2RP included age, gender, performance status, pulmonary fibrosis score, pulmonary function test, smoking history, primary tumor location, clinical stage, concurrent chemotherapeutic regimen, irradiation technique, lung V40, and initial PTV. Univariate analyses revealed that gender (male), pulmonary fibrosis score $(\geq 2)$, smoking history (present), lung V40 ( $\geq 10 \%)$ and initial PTV ( $\geq 398 \mathrm{ml})$ were significant predictor for G2RP (Table 4). There was no difference in the incidence of G2RP between IMRT and 3DCRT. Pulmonary function was also not a predictive factor for G2RP. The variables that showed significance in the univariate analyses were further evaluated in multivariate analyses. In multivariate analyses, pulmonary fibrosis score and lung V40 remained to be significant factors for G2RP ( $p<0.001$, HR: 7.83, 95\%CI: 3.38-18.13, and $p=0.034$, HR: $3.17,95 \%$ CI: 1.09-9.19, respectively). The cumulative incidence of G2RP at 6 months was $16.7 \%$ and $57.3 \%$ with lung V40 of below and above the threshold level of 10\%, respectively (Fig. 5).

\section{Univariate and multivariate analyses of factors affecting risk of eternal discontinuation of durvalumab due to RP}

The variables showed some significance for predicting G2RP were included in the univariate analyses. Univariate analyses revealed that gender (male), pulmonary fibrosis score $(\geq 2)$ and initial PTV $(\geq 398 \mathrm{ml})$ were significant factors for eternal discontinuation of durvalumab (Table 4). There was also no difference in the incidence between IMRT and 3DCRT. The variables that showed significance in the univariate analyses were further evaluated in multivariate analysis. In multivariate analysis, only pulmonary fibrosis score remained to be a significant factor (< 0.001, HR: 5.89, 95\% CI: 1.53-22.68).

\section{Cumulative incidence of G2RP according to lung V20 level}

The 6 months-cumulative incidence of G2RP among patients with lung V20 of $<20 \%, 20-25 \%$ and V20 $\geq 25 \%$ were $25.0,46.7$ and $51.8 \%$ respectively (Fig. 6). There were no statistical differences among them $(p=0.51)$.

\section{Discussion}

In the current study, the incidence of G2RP was 39.3\% after CCRT followed by durvalumab for LA-NSCLC. The incidence seems to be higher than the previous reports without durvalumab [26-29]. From recent 
Table 4 Univariate and multivariate analyses of factors affecting risk of radiation pneumonitis of Grade 2 or more and discontinuation of durvalumab Univariate analysis

\begin{tabular}{|c|c|c|c|c|c|c|c|}
\hline Variables & & $N$ & $\begin{array}{l}6 \mathrm{M} \text { cumulative } \\
\text { incidence of G2RP } \\
(\%)\end{array}$ & $p$ value & $\begin{array}{l}6 \mathrm{M} \text { cumulative incidence of } \\
\text { discontinuation of Durvalumab due to } \\
\mathrm{G} 2 \mathrm{RP}(\%)\end{array}$ & $p$ value & \\
\hline \multirow[t]{2}{*}{ Gender } & Male & 37 & 48.7 & 0.038 & 21.6 & 0.03 & \\
\hline & Female & 19 & 18.6 & & 0.0 & & \\
\hline \multirow[t]{2}{*}{ Pulmonary Fibrosis Score } & $\geq 2$ & 10 & 90.0 & $<0.001$ & 60.0 & $<0.001$ & \\
\hline & $0-1$ & 46 & 28.3 & & 4.3 & & \\
\hline \multirow[t]{2}{*}{ Smoking history } & Present & 45 & 46.5 & 0.031 & 17.8 & 0.14 & \\
\hline & Never & 11 & 9.0 & & 0.0 & & \\
\hline \multirow[t]{2}{*}{ Lung V40 } & $\geq 10 \%$ & 30 & 57.3 & 0.011 & 20.0 & 0.21 & \\
\hline & $<10 \%$ & 26 & 16.7 & & 7.7 & & \\
\hline \multirow[t]{2}{*}{ Initial PTV } & $\geq 398 \mathrm{ml}$ & 24 & 58.3 & 0.024 & 25.0 & 0.048 & \\
\hline & $<398 \mathrm{ml}$ & 32 & 23.8 & & 6.3 & & \\
\hline \multicolumn{8}{|l|}{ Multivariate analysis } \\
\hline \multicolumn{2}{|l|}{ Variables } & HR & $95 \% \mathrm{Cl}$ & $p$ value & HR & $95 \% \mathrm{Cl}$ & $p$ value \\
\hline Pulmonary Fibrosis Score & $\geq 2$ & 7.83 & $3.38-18.13$ & $<0.001$ & 5.89 & $1.53-22.68$ & $<0.001$ \\
\hline Lung V40 & $\geq 10 \%$ & 3.17 & 1.09-9.19 & 0.034 & & & \\
\hline InitialPTV & & & & & 2.62 & $0.71-9.71$ & 0.15 \\
\hline
\end{tabular}

Lung V40 percentage of the lung volume exceeding $x$ Gy, Lung Vs5 volume of the lung receivedless than 5 Gy, HR hazard ratio, PTV planning target volume

multi-institutional retrospective analysis in Japan, the incidence of $24 \%$ for G2RP were reported before introduction of durvalumab [29]. Few reports had reported the real-world incidence of G2RP when durvalumab is administered after CCRT. In coincidence with the current study, reports from several institutions revealed

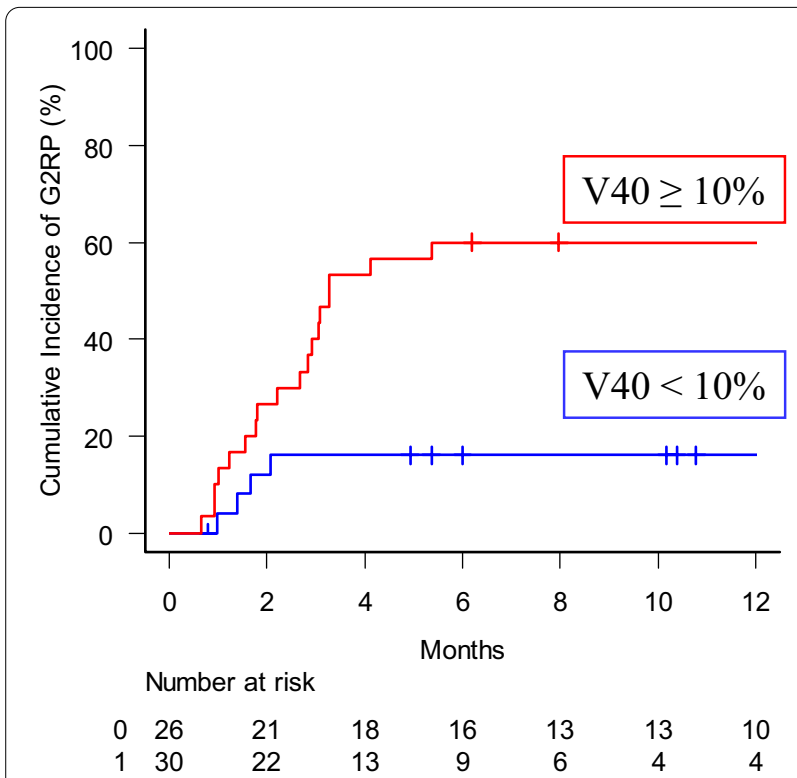

Fig. 5 Cumulative incidence of radiation pneumonitis of grade 2 or more stratified by lung $\mathbf{V} 40$. The dichotomizing value was based on ROC analysis. Lung V40: percentage of lung volume exceeding $40 \mathrm{~Gy}$ the incidence to be 36-43\% [30-32]. Jung et al reported the higher incidence of G2RP among patients administered durvalumab, compared to observed patients (42.9\% vs. $20 \%$ ) [31]. They also reported the higher incidence of Grade 3 RP in the patients with durvalumab, compared to observation ( $14.3 \%$ vs. $2.5 \%)$. Recently reported multi-institutional study in Japan revealed that the incidence of G2RP were $37.7 \%$ with durvalumab [32]. Consolidation with durvalumab should increase the incidence of symptomatic RP, especially in Asian ethnicity patients.

In the consideration for durvalumab, development of G2RP is a clinically important endpoint. One

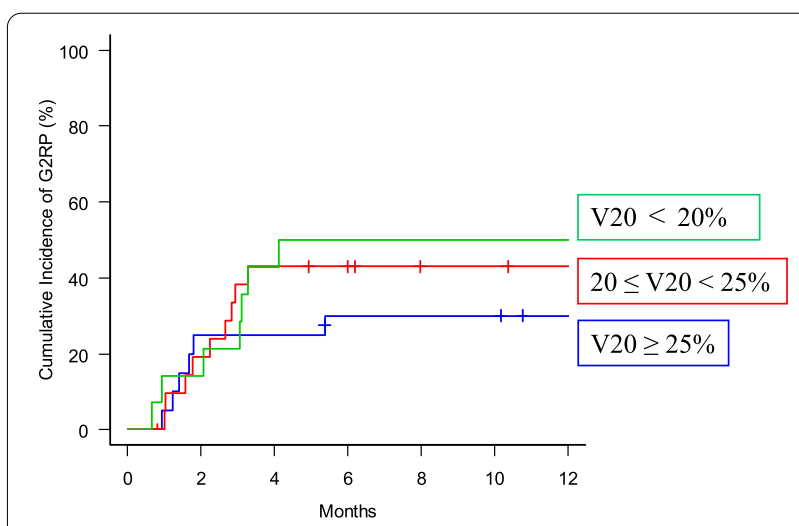

Fig. 6 Cumulative incidence of radiation pneumonitis of grade 2 or more according to lung V20 level. Lung V20: percentage of lung volume exceeding $20 \mathrm{~Gy}$ 
of frequent reasons for discontinuing durvalumab is symptomatic RP. When a patient develops G2RP, durvalumab is interrupted and typically be treated by corticosteroid therapy. Interruption of durvalumab as well as immunologic inhibition by corticosteroid may impair the anti-tumor activity by $\mathrm{T}$ cells, which has been enhanced by durvalumab. Therefore, prediction and prevention of G2RP is crucial.

Dosimetric analysis of this study showed that the percentage of the lung irradiated exceeding 40Gy (V40) were independent predictors for G2RP. Various predicting factors for RP were reported so far [27, 28, 33-35]. Among them, lung doses have been regarded as the most distinct predicting factor for RP. In 2003, Tsujino et al. reported the relationships between lung V20 and the incidence of G2RP in CCRT for LA-NSCLC [28]. The lung V20 of higher than $25 \%$ significantly increased the incidence of G2RP [28]. To reduce the incidence of RP, introduction of new irradiation techniques, such as IMRT or respiratory motion management would be useful [36-38]. However, there is another concern about the risk of excessive low dose irradiation to the lung from the reports of post extrapleural pneumonectomy radiotherapy for pleural mesothelioma $[39,40]$. In the case with CCRT using IMRT for LA-NSCLC, the incidence of G3 RP significantly increased when lung V5 exceeded more than 70\% [41]. In this study, there was no difference in the incidence of Grade 2 or 3 RP between patients who received IMRT or 3D-CRT, and neither V20 nor V5 was significant predictor for G2RP. In contrast to previous reports, in the current study, the volume of the lung irradiated to high dose (V40) found to be independent significant predictors of G2RP. Some previous reports declared the high dose constraint, however, it had not often been highlighted in recent reports [42, 43]. Both of lung V20 and V5 were strictly restricted in the treatment planning in our general practice, irrespective of the irradiation techniques. On the other hands, we did not restrict lung V30 to 50. Possible reason for the correlation between lung $\mathrm{V} 40$ and RP in our cohort might be that the variations in lung V40 were larger than that of V20 and V5. Recently, in line with the current study, Saito et al suggested significant association of medium to high dose-volumes of the lung and G2RP in patients with LA-NSCLC treated with CCRT followed by durvalumab [30]. High dose volumes to the lung should also be associated with the incidence of G2RP in patients with LA-NSCLC treated with CCRT followed by durvalumab. Every effort should be practiced to reduce the high dose irradiated volume of the lung.

In the current study, baseline existence of pulmonary fibrosis was the strongest predictor of G2RP and only an independent predictor of permanent discontinuation of durvalumab due to RP. Association between subclinical interstitial lung disease and fatal radiation pneumonitis was described in several reports [44-46]. Tsujino et al advocated the predictive risk score including subclinical interstitial lung disease for Grade 3 RP [26]. Pulmonary fibrosis score of 2 or more, which has honeycoming, was an independent predictor for Grade 3 RP. When pulmonary fibrosis was scored in combination with another predictors (age $\geq 68$, lung V $20 \geq 26 \%$ and lung Vs5 $<1500 \mathrm{cc})$, the predictability for Grade 3 RP was significantly improved. Taking into consideration of this predictive risk sore in the treatment planning for LA-NSCLC, the incidences of Grade 3 or higher RP radically reduced over time in their institution (personal communication). Careful patient selection for durvalumab is crucial especially for patients who are suspected to have subclinical interstitial lung disease.

Preliminary results of OS and PFS of the current study seems to be comparable to the initial report of the PACIFIC study [18]. However, there were non-negligible difference in the baseline characteristics of the included patients exists between the PACIFC study and the current study. Our study included relatively older patients, with median age of 72 , compared to 64 in the PACIFIC study. Additionally, more unfavorable patients, 10 patients (18\%) with clinical stage IIIC were included in this study, who were not included in the PACIFIC study. These difference in the patient's background might increase the incidents of RP. Regardless of the considerable patient selection biases, preliminary survival outcomes of the current study were similar to that of the PACIFIC study. The results of the current study suggested the reproduced survival benefit of durvalumab in a real-world settings.

We know there are several limitations in the current study. Firstly, because of the retrospective nature, patient selection criteria for both of CCRT and durvalumab may vary among attending physicians. Also, the grading of RP which were based on the medical records may have an impact on the interpretation of the results. Secondly, because irradiation technique was determined at the discretion of the attending radiation oncologists, baseline characteristics of the patients who received CCRT with IMRT or 3D-CRT were not matched with each other. Thirdly, the optimal cut-off value of the lung dose-volume still needs to be investigated because of the limitation in the patient number included in the current study. Lastly, possible biomarkers that may predict the incidence or severity of RP were not investigated in the current study, although a part of the patients' serums were sequentially cryopreserved for future assays under obtained informed consent. We also conducted a multi-institutional prospective clinical trial, WJOG12019L (UMIN000038366) 
and is currently ongoing to investigate efficacy and safety of CCRT using IMRT followed by durvalumab for LA-NSCLC.

\section{Conclusions}

Pulmonary fibrosis sore and lung V40 were significant predictive factors for symptomatic RP in patients with LA-NSCLC after CCRT followed by durvalumab. We should be cautious about the administration of durvalumab for patients having subclinical pulmonary fibrosis. To our best knowledge, this is one of the first report showing the predictive value of high dose volumes to the lung in patients with LA-NSCLC who received CCRT followed by durvalumab.

\begin{abstract}
Abbreviations
CCRT: Concurrent chemoradiotherapy; LA-NSCLC: Locally-advanced nonsmall cell carcinoma; RP: Radiation pneumonitis; $V x$ : Percentage of total lung volume exceeding $x$ Gy; HR: Hazard ratio; $\mathrm{Cl}$ : Confidence interval; OS: Overall survival; IgG: Immunoglobulin G; PD-L1: Programmed death ligand-1; PD-1: Programmed cell death-1; CD80: Cluster of differentiation 80; PFS: Progression-free survival; IMRT: Intensity-modulated radiation therapy; 3D-CRT : 3D-conformal radiation therapy; CT: Computed tomography; MRI: Magnetic resonance imaging; G2RP: Grade 2 or higher radiation pneumonitis; TTDD: Time to discontinue durvalumab; MLD: Mean lung dose; Vs5: Volume of the lung received less than 5 Gy; PTV: Planning target volume; ROC: Receiveroperating characteristic; AUC: Areas under the curve; 4-DCT: Four-dimensional computed tomography; OBI: On-board imager; CBCT: Cone beam computed tomography; GTV: Gross target volume; ITV: Internal target volume; CTV: Clinical target volume; PTX: Paclitaxel; CDDP: Cisplatin; WJOG: West Japan Oncology Group.
\end{abstract}

\section{Supplementary Information}

The online version contains supplementary material available at https://doi. org/10.1186/s13014-021-01979-z.

Additional file 1. Appendix 1: A case presentation on typical clinical course of radiation pneumonitis in a patient with high lung $V 40$ value. Appendix 2: A case presentation on typical clinical course of radiation pneumonitis in a patient with subclinical lung fibrosis.

\section{Acknowledgements}

The authors thank Amerian Journal Experts (https://www.aje.com/) for English language editing.

\section{Authors' contributions}

$\mathrm{HM}, \mathrm{KU}, \mathrm{AH}, \mathrm{HK}$ and $\mathrm{AH}$ were responsible for the study design. $\mathrm{HM}, \mathrm{AH}, \mathrm{KK}, \mathrm{TM}$, $\mathrm{SM}, \mathrm{TI}, \mathrm{HK}, \mathrm{HK}, \mathrm{HO}, \mathrm{TN}, \mathrm{CS}$ and $\mathrm{AH}$ were responsible for the clinical data collection. $\mathrm{HM}, \mathrm{KU}$ and $\mathrm{AH}$ were responsible for the statistical data analysis. $\mathrm{HM}$ was the main contributor for the writing of the manuscript. All authors read and approved the final manuscript.

\section{Funding}

No funding was received for the research reported herein.

\section{Availability of data and materials}

Research data are stored in an institutional repository and will be shared upon request to the corresponding author.

\section{Declarations}

\section{Ethics approval and consent to participate}

The study protocol was approved by the Research Ethics Committee of our institution (reference number: 2020-[study05]-03). All study participants provided informed written consent for therapy. Because of the retrospective nature of this study, individual consent for study inclusion was exempted.

\section{Consent for publication}

Not applicable.

\section{Competing interests}

The authors declare that they have no competing interests.

\section{Author details}

'Department of Radiation Oncology, Kobe Minimally-invasive Cancer Center, 8-5-1, Minatojima-Nakamachi, Chuo-Ku, Kobe, Hyogo 650-0046, Japan. ${ }^{2}$ Division of Radiation Oncology, Kobe University Graduate School of Medicine, 7-5-2, Kusunoki-Cho, Chuo-Ku, Kobe, Hyogo, Japan. ${ }^{3}$ Department of Respiratory Medical Oncology, Kobe Minimally-invasive Cancer Center, 8-5-1, Minatojima-Nakamachi, Chuo-Ku, Kobe, Hyogo, Japan. ${ }^{4}$ Department of Diagnostic Radiology, Kobe Minimally-invasive Cancer Center, 8-5-1, Minatojima-Nakamachi, Chuo-Ku, Kobe, Hyogo, Japan.

Received: 24 August 2021 Accepted: 28 December 2021

Published online: 15 January 2022

\section{References}

1. Global Burden of Disease Cancer Collaboration, Fitzmaurice C, Allen C, Barber RM, et al. Global, regional, and national cancer incidence, mortality, years of life lost, years lived with disability, and disability-adjusted life-years for 32 cancer groups, 1990 to 2015: a systematic analysis for the Global Burden of Disease Study. JAMA Oncol. 2017;3:524-48.

2. Toyoda Y, Tabuchi T, Hama H, at al. Trends in clinical stage distribution and screening detection of cancer in Osaka, Japan: Stomach, colorectum, lung, breast and cervix. PLoS One. 2020;15:e0244644.

3. Bezjak A, Temin S, Franklin G, et al. Definitive and adjuvant radiotherapy in locally advanced non-small-cell lung cancer: American society of clinical oncology clinical practice guideline endorsement of the American Society for Radiation Oncology evidence-based clinical practice guideline. J Clin Oncol. 2015;33:2100-5.

4. Eberhardt WE, De Ruysscher D, Weder W, et al. 2nd ESMO consensus conference in lung cancer: locally advanced stage III non-small-cell lung cancer. Ann Oncol. 2015;26:1573-88.

5. Furuse K, Fukuoka M, Kawahara M, et al. Phase III study of concurrent versus sequential thoracic radiotherapy in combination with mitomycin, vindesine, and cisplatin in unresectable stage III non-small-cell lung cancer. J Clin Oncol. 1999:17:2692-9.

6. Yamamoto N, Nakagawa K, Nishimura Y, et al. Phase III study comparing second- and third-generation regimens with concurrent thoracic radiotherapy in patients with unresectable stage III non-small-cell lung cancer: West Japan Thoracic Oncology Group WJTOG0105. J Clin Oncol. 2010;28:3739-45.

7. Curran WJ, Paulus R, Langer CJ et al. Sequential vs. concurrent chemoradiation for stage III non-small cell lung cancer: randomized phase III trial RTOG 9410. J Natl Cancer Inst. 2011;103:1452-60

8. Bradley JD, Paulus R, Komaki R, et al. Standard-dose versus high-dose conformal radiotherapy with concurrent and consolidation carboplatin plus paclitaxel with or without cetuximab for patients with stage IIIA or IIIB non-small-cell lung cancer (RTOG 0617): a randomised, twoby-two factorial phase 3 study. Lancet Oncol. 2015;16:187-99.

9. Bradley JD, Hu C, Komaki RR, et al. Long-term results of NRG Oncology RTOG 0617: standard-versus high-dose chemoradiotherapy with or without cetuximab for unresectable stage III non-small-cell lung Cancer. J Clin Oncol. 2020;38:706-14.

10. Yoon SM, Shaikh T, Hallman M. Therapeutic management options for stage III non-small cell lung cancer. World J Clin Oncol. 2017;8:1-20. 
11. Segawa Y, Kiura K, Takigawa N, et al. Phase III trial comparing docetaxel and cisplatin combination chemotherapy with mitomycin, vindesine, and cisplatin combination chemotherapy with concurrent thoracic radiotherapy in locally advanced non-small-cell lung cancer: OLCSG 0007. J Clin Oncol. 2010;28:3299-306.

12. Senan S, Brade A, Wang LH, et al. PROCLAIM: randomized phase III trial of pemetrexed-cisplatin or etoposide-cisplatin plus thoracic radiation therapy followed by consolidation chemotherapy in locally advanced nonsquamous non-small-cell lung cancer. J Clin Oncol. 2016;34:953-62.

13. Hanna N, Neubauer M, Yiannoutsos C, et al. Phase III study of cisplatin, etoposide, and concurrent chest radiation with or without consolidation docetaxel in patients with inoperable stage III nonsmall-cell lung cancer: the Hoosier Oncology Group and U.S. Oncology. J Clin Oncol 2008; 26: 5755-60.

14. Kelly K, Chansky K, Gaspar LE, et al. Phase III trial of maintenance gefitinib or placebo after concurrent chemoradiotherapy and docetaxel consolidation in inoperable stage III non-small-cell lung cancer: SWOG S0023. J Clin Oncol. 2008:26:2450-6.

15. Butts C, Socinski MA, Mitchell PL, et al. Tecemotide (L-BLP25) versus placebo after chemoradiotherapy for stage III nonsmall-cell lung cancer (START): a randomised, double-blind, phase 3 trial. Lancet Oncol. 2014;15:59-68.

16. Ahn JS, Ahn YC, Kim JH, et al. Multinational randomized phase III trial with or without consolidation chemotherapy using docetaxel and cisplatin after concurrent chemoradiation in inoperable stage III non-small-cell lung cancer: KCSG-LU05-04. J Clin Oncol. 2015;33:2660-6.

17. Tsujino K, Kurata T, Yamamoto S, et al. Is consolidation chemotherapy after concurrent chemo-radiotherapy beneficial for patients with locally advanced nonsmall-cell lung cancer? A pooled analysis of the literature. J Thorac Oncol. 2013;8:1181-9.

18. Antonia SJ, Villegas A, Daniel D, Vicente D, Murakami S, Hui R, et al. Durvalumab after chemoradiotherapy in stage III non-small-cell lung cancer. N Engl J Med. 2017;377:1919-29.

19. Antonia SJ, Villegas A, Daniel D, Vicente D, Murakami S, Hui R, et al. Overall survival with Durvalumab after chemoradiotherapy in stage III NSCLC. N Engl J Med. 2018;379:2342-50.

20. Faivre-Finn C, Vicente D, Kurata T, et al. Four-year survival with durvalumab after chemoradiotherapy in stage III NSCLC - an update from the PACIFIC trial. J Thorac Oncol. 2021;16:860-7.

21. Stewart R, Morrow M, Hammond SA, et al. Identification and characterization of MEDI4736, an antagonistic anti-PD-L1 monoclonal antibody. Cancer Immunol Res. 2015;3:1052-62.

22. Pardoll DM. The blockade of immune checkpoints in cancer immunotherapy. Nat Rev Cancer. 2012;12:252-64.

23. Postow MA, Callahan MK, Wolchok JD. Immune checkpoint blockade in cancer therapy. J Clin Oncol. 2015;33:1974-82.

24. Ettinger DS, Wood DE, Aisner DL, et al. NCCN guidelines insights: non-small cell lung cancer, version 2.2021. J Natl Compr Canc Netw. 2021;19:254-266.

25. Kazerooni EA, Martinez FJ, Flint A, et al. Thin-section CT obtained at 10-mm increments versus limited three-level thin-section CT for idiopathic pulmonary fibrosis: correlation with pathologic scoring. AJR Am J Roentgenol. 1997;169:977-83.

26. Tsujino K, Hashimoto T, Shimada T, et al. Combined analysis of V20, VS5, pulmonary fibrosis score on baseline computed tomography, and patient age improves prediction of severe radiation pneumonitis after concurrent chemoradiotherapy for locally advanced non-small-cell lung cancer. J Thorac Oncol. 2014;9:983-90.

27. Graham MV, Purdy JA, Emami B, et al. Clinical dose-volume histogram analysis for pneumonitis after 3D treatment for non-small cell lung cancer (NSCLC). Int J Radiat Oncol Biol Phys. 1999;45:323-9.

28. Tsujino $K$, Hirota $S$, Endo $M$, et al. Predictive value of dose-volume histogram parameters for predicting radiation pneumonitis after concurrent chemoradiation for lung cancer. Int J Radiat Oncol Biol Phys. 2003;55:110-5.

29. Horinouchi H, Atagi S, Oizumi S, et al. Real-world outcomes of chemoradiotherapy for unresectable Stage III non-small cell lung cancer: the SOLUTION study. Cancer Med. 2020;9:6597-608.

30. Saito S, Abe T, Kobayashi N, et al. Incidence and dose-volume relationship of radiation pneumonitis after concurrent chemoradiotherapy followed by durvalumab for locally advanced non-small cell lung cancer. Clin Transl Radiat Oncol. 2020;23:85-8.

31. Jung HA, Noh JM, Sun J-M, et al. Real world data of durvalumab consolidation after chemoradiotherapy in stage III non-small-cell lung cancer. Lung Cancer. 2020;146:23-9.

32. Shintani T, Kishi N, Matsuo T et al. Incidence and risk factors of symptomatic radiation pneumonitis in non-small-cell lung cancer patients treated with concurrent chemoradiotherapy and consolidation Durvalumab. Clin Lung Cancer. 2021;22:401-410.

33. Mehta $\mathrm{V}$. Radiation pneumonitis and pulmonary fibrosis in non-small-cell lung cancer: pulmonary function, prediction, and prevention. Int J Radiat Oncol Biol Phys. 2005;63:5-24.

34. Marks LB, Bentzen SM, Deasy JO, et al. Radiation dose-volume effects in the lung. Int J Radiat Oncol Biol Phys. 2010;76(3 Suppl):S70-6.

35. Kong FS, Wang S, et al. Nondosimetric risk factors for radiation-induced lung toxicity. Semin Radiat Oncol. 2015;25:100-9.

36. Liao ZX, Komaki RR, Thames HD, et al. Influence of technologic advances on outcomes in patients with unresectable, locally advanced non-smallcell lung cancer receiving concomitant chemoradiotherapy. Int J Radiat Oncol Biol Phys. 2010;76:775-81.

37. Hu X, He W, Wen S, et al. Is IMRT superior or inferior to 3DCRT in radiotherapy for NSCLC? A meta-analysis PLoS One. 2016;11:e0151988.

38. Boyle J, Ackerson B, Gu L, Kelsey CR. Dosimetric advantages of intensity modulated radiation therapy in locally advanced lung cancer. Adv Radiat Oncol. 2017;2:6-11.

39. Allen AM, Czerminska M, Jänne PA, et al. Fatal pneumonitis associated with intensity-modulated radiation therapy for mesothelioma. Int J Radiat Oncol Biol Phys. 2006;65:640-5.

40. Chi A, Liao Z, Nguyen NP, et al. Intensity-modulated radiotherapy after extrapleural pneumonectomy in the combined-modality treatment of malignant pleural mesothelioma. J Thorac Oncol. 2011;6:1132-41.

41. Yom SS, Liao Z, Liu HH, et al. Initial evaluation of treatment-related pneumonitis in advanced-stage non-small-cell lung cancer patients treated with concurrent chemotherapy and intensity-modulated radiotherapy. Int J Radiat Oncol Biol Phys. 2007;68:94-102.

42. Jin H, Tucker SL, Liu HH, et al. Dose-volume thresholds and smoking status for the risk of treatment-related pneumonitis in inoperable non-small cell lung cancer treated with definitive radiotherapy. Radiother Oncol. 2009;91:427-32.

43. Harris WB, Zou W, Cheng C, et al. Higher dose volumes may be better for evaluating radiation pneumonitis in lung proton therapy patients compared with traditional photon-based dose constraints. Adv Radiat Oncol. 2020;29(5):943-50.

44. Yamaguchi S, Ohguri T, Matsuki Y, et al. Radiotherapy for thoracic tumors: association between subclinical interstitial lung disease and fatal radiation pneumonitis. Int J Clin Oncol. 2015;20:45-52.

45. Lee YH, Kim YS, Lee SN, et al. Interstitial lung change in pre-radiation therapy computed tomography is a risk factor for severe radiation pneumonitis. Cancer Res Treat. 2015;47:676-86.

46. Niska JR, Schild SE, Rule WG, et al. Fatal radiation pneumonitis in patients with subclinical interstitial lung disease. Clin Lung Cancer. 2018;19:e417-20.

\section{Publisher's Note}

Springer Nature remains neutral with regard to jurisdictional claims in published maps and institutional affiliations. 